\title{
Modeling Using 1-D Map of Complex Behavior in Coupled Chaotic Circuits with Intermittency
}

\author{
Yoko Uwate* ${ }^{*} \quad$ Yoshifumi Nishio *
}

\begin{abstract}
In our previous research, a complex behavior in two coupled chaotic circuits related with intermittency has been investigated. This paper provides modeling of the complex behavior using 1D map derived from the subcircuit and occurrence probabilities of different states. The 1-D map is shown to be useful to make clear the statistical properties of laminar parts. Further, the basin of initial values attracted to the different states is shown to give the information of the occurrence probabilities of the states.
\end{abstract}

\section{INTRODUCTION}

Coupled chaotic circuits systems are good models to describe various higher-dimensional nonlinear phenomena in the field of natural science. In particular, the chaos synchronization and the breakdown of chaos synchronization have attracted many researchers' attentions and their mechanisms have been gradually made clear [1]-[5]. Furthermore, many researchers consider that complex behavior around chaos synchronization in coupled oscillatory circuits are related to information processing of the brain. We consider that it is very important to investigate complex behavior around chaos synchronization for future engineering applications to realize "brain computer."

On the other hand, intermittency chaos is very interested phenomena [6][7]. Because the intermittency chaos, which has stability and mobility, gains good performance for various kind of information processing. We consider that a lot of phenomena in the world are related to the intermittency chaos. For example, information processing of the brain, vegetation in the Arizona Desert and so on. In order to make clear the mechanism of such phenomena in the world, unveiling the roles of intermittency chaos is very important.

In our previous research, a complex behavior in two coupled chaotic circuits related with intermittency has been investigated [8]. When we set a control parameter of each chaotic circuit to generate intermittency chaos near the three-periodic window, we have observed a complex behavior such that intermittency bursts interrupt synchronizations and

\footnotetext{
*Department of Electrical and Electronic Engineering, Tokushima University, JAPAN, 2-1 MinamiJosanjima, Tokushima 770-8506, Japan. Email: \{uwate, nishio\}@ee.tokushima-u.ac.jp, Tel: +81-88-656-7470, Fax: +81-88-656-7471.
}

different synchronizations reappear after the bursts settle down. We have modeled this interesting complex behavior by a first-order Markov chain with four states and have confirmed the results obtained from the Markov chain agree very well with computer simulated results [8]. However, in the modeling by the Markov chain, the transition probabilities between the states are obtained by counting all of the transitions in plenty of computer simulation. Hence, the Markov chain model needs vast amounts of calculations when the coupled circuits system becomes very large.

In this study, we pay our attentions to that the switching of the synchronization modes in the complex behavior is caused by intermittency bursts of each chaotic circuit. If we know the timing of generating bursts in one chaotic circuit, the statistical properties of the complex behavior could be explained. Hence, we derive the 1-D Poincaré map from one chaotic circuit. By investigating this 1D map, we predict the statistical property of the complex behavior generated in the coupled chaotic circuits. By computer simulations, the predicted average length of the laminar part is confirmed to agree with that observed in the coupled chaotic circuits. Further, the occurrence probabilities of the different states are obtained from the basin of initial values for the states.

\section{CIRCUIT MODEL}

Figure 1 shows the circuit model, which is the asymmetric version of the circuit investigated in [9]. In the circuit, two identical chaotic circuits are coupled by a resistor $R$.

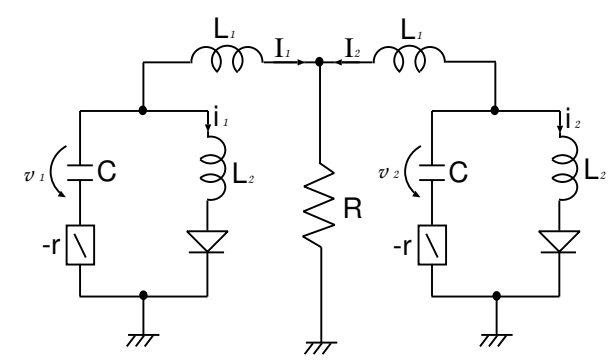

Figure 1: Circuit model.

At first, the $i-v$ characteristics of the diodes are approximated by two-segment piecewise-linear 
functions as

$$
v_{d}\left(i_{k}\right)=0.5\left(r_{d} i_{k}+E-\left|r_{d} i_{k}-E\right|\right) .
$$

By changing the variables and parameters,

$$
\begin{gathered}
I_{k}=\sqrt{\frac{C}{L_{1}}} E x_{k}, \quad i_{k}=\sqrt{\frac{C}{L_{1}}} E y_{k}, \quad v_{k}=E z_{k}, \\
t=\sqrt{L_{1} C} \tau, \quad \alpha=\frac{L_{1}}{L_{2}}, \quad \beta=r \sqrt{\frac{C}{L_{1}}}, \\
\gamma=R \sqrt{\frac{C}{L_{1}}}, \quad \delta=r_{d} \sqrt{\frac{C}{L_{1}}}
\end{gathered}
$$

the normalized circuit equations are given as

$$
\left\{\begin{array}{l}
\frac{d x_{k}}{d \tau}=\beta\left(x_{k}+y_{k}\right)-z_{k}-\gamma\left(x_{1}+x_{2}\right) \\
\frac{d y_{k}}{d \tau}=\alpha \beta\left(x_{k}+y_{k}\right)-z_{k}-f\left(y_{k}\right) \\
\frac{d z_{k}}{d \tau}=x_{k}+y_{k} \quad(k=1,2)
\end{array}\right.
$$

where

$$
f\left(y_{k}\right)=0.5\left(\delta y_{k}+1-\left|\delta y_{k}-1\right|\right) .
$$

\section{COMPLEX BEHAVIOR}

Figure 2 shows three different types of synchronization states, when the two circuits generate threeperiodic attractors. These three synchronization states can be obtained by giving different initial conditions. As we can see from the figures, the two circuits tend to be synchronized in anti-phase. This is because the circuits tend to minimize the energy consumed by the coupling resistor $R$. There exist three different peaks in the waveform of the threeperiodic solution. Hence, three different synchronization states could coexist as shown in Fig. 2 . We name the three synchronization states as the states $T_{1}, T_{2}$, and $T_{3}$.
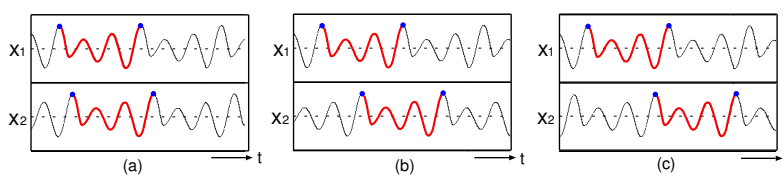

Figure 2: Time waveforms of three synchronization states (computer calculated results). $\alpha=7.0, \beta=$ $0.152, \gamma=0.005$ and $\delta=100.0$. (a) State $T_{1}$, (b) State $T_{2}$, and (c) State $T_{3}$.

Next, we vary a control parameter of each chaotic circuit to generate intermittency chaos near the three-periodic window as shown in Fig. 3. In this case, we can observe a complex behavior of the three synchronization states. Namely, intermittency bursts disturb the synchronizations and different synchronizations reappear after the bursts settle down.
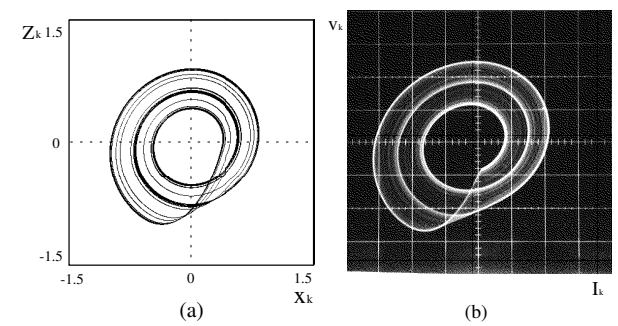

(b)

Figure 3: Intermittency chaos near the threeperiodic window. (a) Computer calculated result. $x_{k}$ vs. $z_{k} . \quad \alpha=7.0, \beta=0.133682, \gamma=0.0$ and $\delta=100.0$. (b) Circuit experimental result. $I_{k}$ vs. $v_{k} . L_{1}=300 \mathrm{mH}, L_{2}=10 \mathrm{mH}, C=33 \mathrm{nF}, r=735 \Omega$ and $R=0.0 \Omega$.

Figure 4 shows how the three states appear and disappear in a chaotic way. This diagram shows the discrete data of $x_{2}$ only on the cross section $z_{1}=0$ and $x_{1}<-1.2$, namely, the value of $x_{2}$ only when the $x_{1}$ takes the largest valley in Fig. 2. We also confirmed the same phenomenon in the circuit experiments [8].

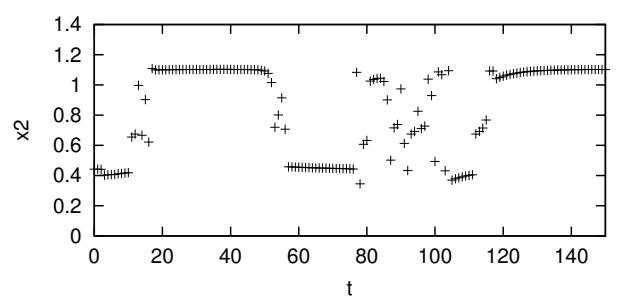

Figure 4: Time series of synchronization states disturbed by intermittency chaos (computer calculated results). $\alpha=7.0, \beta=0.133682, \gamma=0.005$ and $\delta=100.0$.

\section{DERIVATION OF 1-D MAP}

We consider that the switching of the synchronization states in the complex behavior is caused by intermittency bursts of each chaotic circuit. Hence, if we know the timing of generating bursts in one chaotic circuit, the statistical properties of the complex behavior could be explained. In this study, we model the interesting complex behavior by using 1-D Poincaré map derived from one chaotic circuit.

We consider the subcircuit of the circuit model in Fig. 1. We define the Poincaré section as $z=0$ and $x<0$. Since $y$, which is corresponding to the current through $L_{2}$, is almost zero for $x<0$ because of the switching characteristics of the diode, the Poincaré map can be described by only the value of $x$ approximately. Namely, the Poincaré map of the subcircuit can be derived as a 1-D map. The obtained 1-D map is shown in Fig. 5. Further, the 
time series of intermittency chaos obtained from the 1-D map is shown in Fig. 6.

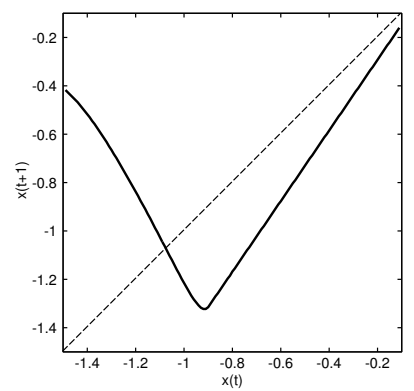

Figure 5: One-dimentional Poincaré map of subcircuit. $\alpha=7.0, \beta=0.133682, \gamma=0.0045$ and $\delta=100.0$.

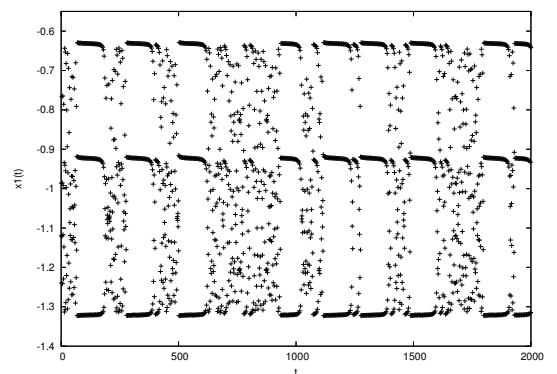

Figure 6: Time series of intermittency chaos in 1D map. $\alpha=7.0, \beta=0.133682, \gamma=0.0045$ and $\delta=100.0$.

\section{ANALYSIS OF 1-D MAP}

In order to model the complex behavior observed in the coupled chaotic circuits, we investigate the time series obtained from the 1-D map in detail.

At first, we distinguish laminar parts and burst parts of the intermittency chaos. Because we treat only the intermittency chaos near the threeperiodic window, we regard three successive sequences starting from a point whose value is -0.64 or larger as one-period of the laminar part (see Fig. 6). Other points are regarded as the burst part. Next, we count the number of generating periods of the laminar parts. The probability of each period of the laminar parts during 21000 iterations of the 1-D map is shown in Fig. 7(a). We can see that the curve does not obey any simple scaling rules. Namely, the period of the laminar part is bounded and the maximum value of the period takes a peak. We consider that this is the most distinguished feature of the intermittency chaos of the 1-D map.

In our supposition, if the laminar part changes to the burst part in one subcircuit, the other subcircuit is also drawn into the burst part. In order to

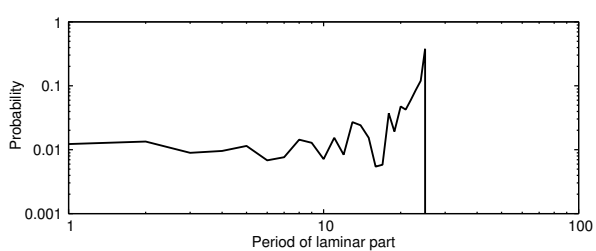

(a) $P(k)$ for 1-D map.

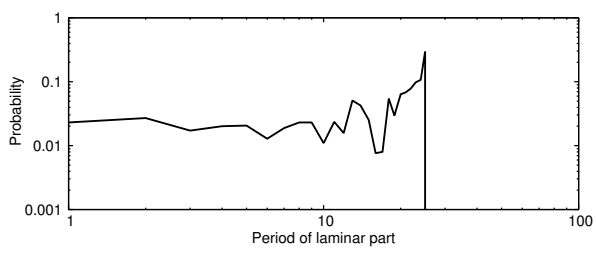

(b) $\hat{P}(k)$ for two 1-D maps.

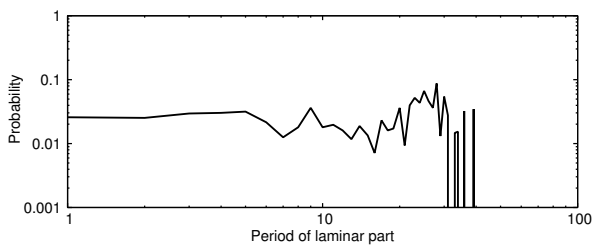

(c) Probability obtained from coupled circuits.

Figure 7: Probability distribution of periods of laminar parts.

realize this effect, we have to consider a new probability function using two 1-D maps. The probability function $\hat{P}(k)$ corresponding to the coupled chaotic circuits is defined by the following equations,

$$
\left\{\begin{array}{l}
\hat{P}(1)=2 P(1) \\
\hat{P}(k)=2 P(k)\left(1-\sum_{i=1}^{k-1} P(i)\right) \quad(k=2 \ldots N)
\end{array}\right.
$$

where $P(k)$ denotes the probability of $k$-period of laminar part of the $1-\mathrm{D}$ map and $\hat{P}(k)$ denotes the probability such that the period of the laminar part of one 1-D map is $k$ and the period of the laminar part of the other 1-D map is not less than $k$. Figure $7(\mathrm{~b})$ shows the probability $\hat{P}(k)$ obtained by using the results in Fig. 7(a) and Eq. (5).

In order to confirm that the coupled chaotic circuits have the same statistical property, we count the number of generating periods of the laminar parts in the data obtained from the circuits The probability of each period of the laminar parts during 21000 rotations in the attractor of the circuit. is shown in Fig. 7(c). We can say that the distribution is close to that in Fig. 7(b).

Further, we compare the average length of the laminar parts in the coupled chaotic circuits and that obtained theoretically by using the probability $\hat{P}(k)$ based on the 1-D map. The result is summarized in Tab. 1. We can confirm that the average 
length of the laminar parts obtained by using the 1D map is similar to that obtained from the original coupled chaotic circuits. Namely, we can say that the statistical property of the complex behavior of the coupled chaotic circuits can be modeled by the simple 1-D map derived from one subcircuit.

Table 1: Average length of laminar part.

\begin{tabular}{|c|c|}
\hline Coupled circuits & Two 1-D maps \\
\hline \hline 21.1631 & 21.5204 \\
\hline
\end{tabular}

\section{OCCURRENCE PROBABILITIES OF THREE STATES}

In the previous section, we have shown that the 1D map corresponding to the subcircuit is useful to make clear the statistical properties of the laminar parts of the coupled circuits. However, there exist three different states $T_{1}, T_{2}$ and $T_{3}$ in the laminar parts as shown in Figs. 2 and 4, and the above analysis does not give any information of the occurrence of the three states.

In this section, we consider the occurrence probabilities of the three states. When the circuits are in the burst parts, the values of $x_{1}$ and $x_{2}$ are not correlated. Hence, if we know the probabilities generating the three states when initial values are given at random in a certain region, the probabilities are considered to correspond to the occurrence probabilities of the three states in the complex behavior.

Figure 8 shows the basin of the initial values $x_{1}$ and $x_{2}$ attracted to the three different states $T_{1}, T_{2}$ and $T_{3}$ when $y_{1}=z_{1}=y_{2}=z_{2}$ are set to 0.0 . Because the solution is always around the hyperplane $x_{1}+x_{2}=0$ corresponding to anti-synchronization, the signs of the ranges of $x_{1}$ and $x_{2}$ are not the same.

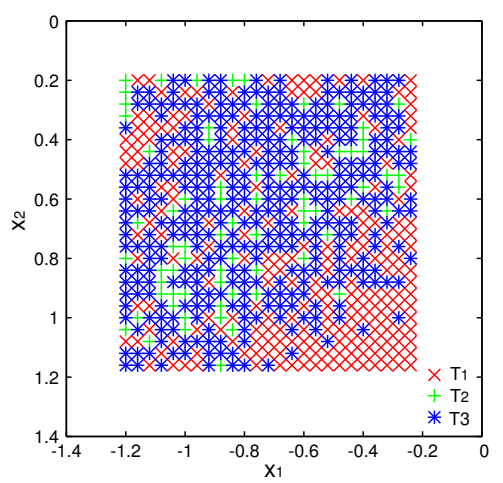

Figure 8: Basin of initial values for three states.

The occurrence probabilities of the three different states are summarized in Tab. 2. In the tables, "Coupled circuits" is the data obtained by counting the states in computer simulations of the circuits and "Space ratio" is the probabilities obtained by using the space ratio in Fig. 8. We can see that the results agree well.

Table 2: Occurrence probabilities.

\begin{tabular}{|c||c|c|}
\hline State & Coupled circuits & Space ratio \\
\hline \hline$T_{1}$ & $\mathbf{0 . 5 5 4 4}$ & $\mathbf{0 . 5 4 4 0}$ \\
\hline$T_{2}$ & $\mathbf{0 . 1 0 0 1}$ & $\mathbf{0 . 1 2 1 6}$ \\
\hline$T_{3}$ & $\mathbf{0 . 3 4 5 5}$ & $\mathbf{0 . 3 3 4 4}$ \\
\hline
\end{tabular}

As a result, we can say that the complex behavior in two coupled chaotic circuits can be modeled by using the 1-D map derived from the subcircuit and the occurrence probabilities of the states.

\section{CONCLUSIONS}

In this study, we have modeled the complex behavior in coupled chaotic circuits with intermittency by using the 1-D map derived from the subcircuit and the occurrence probabilities of the states. The 1-D map has been shown to be useful to make clear the statistical properties of the laminar parts. Further, the basin of the initial values attracted to the different states has been shown to give the information of the occurrence probabilities of the states.

\section{References}

[1] N. Platt, E.A. Spiegel and C. Tresser, "On-Off Intermittency: A Mechanism for Bursting," Phys. Rev. Lett., vol. 70, no. 3, pp. 279-282, 1993

[2] E. Ott and J.C. Sommerer, "Blowout Bifurcations: the Occurrence of Riddled Basins and On-Off Intermittency," Phys. Lett., vol. A188, pp. 39-47, 1994.

[3] P. Ashwin, J. Buescu and I. Stewart, "Bubbling of Attractors and Synchronisation of Chaotic Oscillators," Phys. Lett., vol. A193, pp. 126-139, 1994.

[4] T. Kapitaniak and L.O. Chua, "LocallyIntermingled Basins of Attraction in Coupled Chua's Circuits," Int. J. Bifurcation and Chaos, vol. 6, no. 2, pp. 357-366, 1996.

[5] M. Wada, Y. Nishio and A. Ushida, "Analysis of Bifurcation Phenomena on Two Chaotic Circuits Coupled by an Inductor," IEICE Trans. Fundamentals, vol. E80-A, no. 5, pp. 869-875, May 1997.

[6] Y. Pomeau and P. Manneville, "Intermittent Transition to Turbulence in Dissipative Dynamical Systems," Comm. Math. Phys., vol. 74, pp. 189-197, 1980.

[7] C.G. Langton, "Computation at the Edge of Chaos: Phase Transitions and Emergent Computation," Physica D, vol. 42, pp. 12-37, 1990.

[8] Y. Uwate, Y. Nishio, "Complex Behavior in Coupled Chaotic Circuits Related with Intermittency," Proc. NOLTA'04, pp. 589-592, Nov. 2004.

[9] Y. Nishio, K. Suzuki, S. Mori and A. Ushida, "Synchronization in Mutually Coupled Chaotic Circuits," Proc. ECCTD'93, vol. 1, pp. 637-642, Aug. 1993. 